\title{
Fundamentos filosóficos de las terapias de la atribución
}

\section{Richard Totman}

FufNTF: Ch. Antaki y Ch. Brewin (Eds.), Attributions and Psycbological Cbange. Londres, Academic Press, 1982.

\section{A. INTRODUCCION}

La teoria de la atribución trata de las reflexiones conscientes de las personas sobre sus propias acciones y las de los demás, y las explicaciones que se inventan para justificar estas acciones. La psicologia tiene un mal historial cuando se trata de producir teorias e ideas con valor práctico, y uno de los atractivos de la teoria de la atribución es que parece que se presta a su aplicación práctica en terapia y educación. Este capítulo está escrito con el convencimiento de que la teoría tiene de hecho un papè práctico que jugar en estos y otros campos de la problemática social, pero que para comprender, desarrollar y consolidar este papel es esencial apreciar qué tipo de innovaciones hay en esta teoría y cuáles son sus límites. Al igual que con todas las nuevas propuestas es muy fácil entusiasmarse y sobrevalorar su importancia.

$\mathrm{La}$ teoría (o más bien conjunto de ideas, ya que hay poco de teoría en el sentido estricto) se basa en dos presupuestos básicos, 1) que las personas hacen atribuciones, por ejemplo intentar explicar acciones, y 2) que es posible hacer distinciones y generalizaciones en los tipos de explicaciones que, por lo general, se inventan, esto es, podemos clasificar de forma exhaustiva los tipos de ideas concebidas por la gente para explicar acciones. El primero de estos presupuestos ha sido objeto de ciertas discrepancias y reservas (por ejemplo Langer, 1978). Sin embargo, si no desde la investigación y teoria psicológica, sí desde nuestras observaciones cotidianas, podemos ver con cierta seguridad que los individuos dedican gran parte de su tiempo a dar sentido y evaluar las cosas que otras personas hacen, así como a justificar sus propias acciones. La conversación casual y el cotilleo están llenos de críticas directas e indirectas y (lo que es más raro) dan el visto bueno a las acciones de los demás. Está claro que no sería correcto decir que cada acción de cada individuo es siempre realizada y examinada. La mayor parte de la conducta humana se debe bastante a la casualidad y pasa prácticamente desapercibida. Por lo tanto, ¿cuándo se hacen las atribuciones?, ¿en qué circunstancias considera la 
gente necesario justificar sus propias acciones y explicar lo que los demás hacen? La teoria de la disonancia cognitiva de Festinger (Festinger, 1957) indica que las explicaciones se exigen sobre todo cuando se percibe una acción como fuera de línea con lo que es normal y esperado en esas circunstancias (también Totman, 1973). Como Langer señala «... a menos que se esté obligado a tomar parte con el pensamiento consciente, el modo preferido de interacción con el propio entorno es el de un estado de relativa ausencia de pensamiento, al menos en relación con la situación que se tiene entre manos» (p.40).

El discurso corriente de la gente también apoya esta conclusión. La lógica de la pregunta "ipor qué hizo él eso?», o «por qué lo hice yo?» (una u otra se da siempre en todos los análisis atribucionales como punto de arranque de la actividad cognitiva del sujeto), implica que la necesidad de explicación aparece sólo cuando el individuo se ha planteado la posibilidad de salirse de una regla o forma estándar de normalidad u orden.

El segundo presupuesto, que las explicaciones que las personas ofrecen son en sí susceptibles de explicación, o al menos de clasificarse en categorías teóricamente significativas, es el punto de partida de la mayor parte de la investigación y teoría de la atribución. Por tanto, la teoria de la atribución es en realidad un conjunto de distinciones relacionadas con los tipos de explicación que suelen ofrecerse para referirse a hechos pasados, y un conjunto correspondiente de hipótesis sobre qué es lo que determina la selección de una explicación en una situación y cuạ́l será el efecto de seleccionar un tipo concreto de explicación sobre el carácter, conducta y actitudes de la persona.

\section{B. ATRIBUCIONES INTERNAS Y EXTERNAS}

La distinción entre atribuciones internas y externas juega un papel decisivo en gran parte de los análisis de la teoría de la atribución, y está en el centro de los programas de prevención e intervención de la terapia basada en la atribución. Ya que esta distinción es tan importante, es oportuno considerarla aquí en detalle, volviendo a algunos de los puntos señalados en la introducción y añadiendo otros nuevos: ¿Está basada en una lógica sólida?, ¿tiene sentido contrastar causas percibidas como «internas» de las personas con causas percibidas como uexternas" a ellas?, ¿es esta distinción realmente fundamental o está mezclada con otras?

De hecho, la distinción interna-externa tiene su paralelo en el lenguaje corriente, en la ley y en filosofia, lo cual es esencial no dejarlo de lado. La evolución y el refinamiento de las ideas en la ley y en filosofía constituye un reflejo de los problemas fundamentales del hombre social. La teoría de la atribución no es más que un desarrollo reciente en un campo comparativamente joven. Identificar sus categorias y distinciones en la línea de otras más antiguas es darle un apoyo considerable, al establecer precedentes analíticos que se han gestado, no durante años, sino durante siglos, y que por lo tanto no se pueden desechar como superficiales o resultado de la moda.

\section{Lenguaje corriente}

Yo ya he afirmado en otros escritos (Totman, 1973, 1980, en prensa) que muchas de las tesis más ampliamente discutidas en psicología social se pueden deducir de la lógica y forma del lenguaje corriente de la gente. Sabemos, por ejemplo, que las atribuciones y otras formas de explicación, por lo general, sólo se buscan en circunstancias anómalas que no están resueltas. Los "porqués» son exigencias de explicación, y no surgen en situaciones de cotidianeidad y normalidad. Cuando un niño porfiado, extrañado por la conducta de alguien, se embarca en una de esas interminables cadenas de preguntas, el padre exasperado, al final, no encuentra otra forma de contes- 
tar que con la frase "porque ésas son las cosas que ese tipo de persona hacen». En verdad no necesitamos la teoría de la disonancia cognitiva, e incluso ni de la investigación psicológica, para ser capaces de determinar en qué circunstancias se hacen las atribuciones.

También podemos, partiendo de la intuición y sin demasiada dificultad, clasificar en dos tipos las afirmaciones hechas por los individuos sobre las acciones de otras personas: "cosas hechas a una persona» $y$ "cosas hechas por una persona». La importancia de esta distinción en un análisis sobre los comentarios de un acto/acción es bastante evidente, ya que toda la retórica de la culpabilidad y responsabilidad depende de cuál de éstas es la apropiada. Por definición, no se puede culpabilizar a alguien por algo en lo que no tiene elección.

Pero, ¿qué significado tiene el ver que no hay elección? Ver que no hay elección supone que es evidente que se actúa bajo la influencia de alguna fuerza poderosa. En la medida en que esa influencia coercitiva es visible para el observador, al individuo se le atribuirá poca capacidad de elección y se le dejará exento de la culpabilidad y responsabilidad (o crédito) que podría haberse derivado de su acción, si tal influencia no hubiera sido identificable. Una cierta observación del lenguaje de cada día nos muestra que el torrente de frases relativas a la culpabilidad y responsabilidad dependen estrechamente de una atribución inicial de libertad, o más concretamente, de ausencia total de coacción. Algo muy próximo a la distinción interna-externa de la teoría de la atribución parece basarse en el lenguaje cotidiano.

\section{El sistema legal}

El ejercicio de la justicia en los litigios, como es obvio, se ve también muy afectado por la cuestión de la agencia personal. El señor $A$, que mata de un tiro a uno que ha entrado en su casa para robar y tenía la pistola apoyada en la cabeza de su mujer, será absuelto con más facilidad que el señor $B$, que mata de un tiro a un ladrón sin ninguna excusa de este tipo. El argumento básico es que A tenia menos probabilidades de elección que $B$, ya que se acepta como normal y razonable, e incluso deseable, que sea prioritario el proteger la vida de aquellos que nos son próximos.

Las defensas de los delitos en los juzgados giran en torno a esta cuestión del grado de elección que es apropiado atribuir al acusado. Si puede establecerse que un determinado crimen fue un «crimen pasional», o se cometió «durante una perturbación mental temporal», o incluso si puede establecerse alguna conexión entre una infancia miserable y la conducta por la que se acusa a la persona, entonces la condena puede ser menor. La estratagema, de nuevo, es vender al jurado la idea de que el lugar de control estaba fuera del proceso racional, calculado y volitivo del individuo.

\section{Filosofia}

La distinción entre agencia y pasividad atribuidas, también subyace a algunos de los temas más importantes de la filosofia de la acción; por ejemplo, en el debate sobre razones frente a causas en la explicación de la conducta social humana, y la controversia sobre el libre albedrio frente a la determinación de la acción. Gran parte de esta rama de la filosofia se dedica a tratar la naturaleza de las afirmaciones que la gente hace, y la permanencia de estas controversias refleja la existencia de dos claras e importantes clases de versión explicativa.

Así, pues, hay una cierta correspondencia entre las distinciones básicas para la ley y la filosofia, y las que hemos obtenido al considerar la estructura sintáctica de las afirmaciones que la gente hace, de forma reflexiva, para referirse a los hechos y acciones. Esto tiene que ver con la atribución de la responsabilidad moral y parece estar en íntima relación con la distinción interna-externa de la 
teoria de la atribución. Sin embargo, por desgracia, no puede establecerse un nexo claro entre el concepto de atribución y estos otros más antiguos. Está lejos de ser perfecto el acuerdo entre los atribucionistas sobre lo que se quiere decir con interno-externo. Autores distintos han acentuado aspectos diferentes del proceso de atribución, y esto ha llevado a una serie de definiciones diferentes. Gergen (1978) ha criticado muy acertadamente a los psicólogos sociales por las estrechas perspectivas ahistóricas que suelen adoptar. Los atribucionistas no están exentos de esta crítica. Es un dato contra la teoría de la atribución, el que sus conceptos y distinciones básicos se hayan establecido en base a los experimentos de laboratorio, y que apenas se mencionan formas e instituciones, tales como nuestros sistemas legales y judiciales, que han estado en el mismo corazón de la vida social durante milenios. Tales instituciones constituyen en si cristalizaciones de dimensiones fundamentales, en las que se han llevado a cabo las distinciones más provechosas en la explicación de los actos y acciones humanos. Una de las dimensiones más sobresalientes entre éstas, es la que va desde la (atribuida) acción sin coacción, libremente elegida, de la que el individuo puede ser considerado responsable, a la (atribuida) acción forzada o coaccionada, por la que no se puede culpabilizar al individuo.

\section{LA TEORIA DE LA ATRIBUCION COMO ANTIEMPIRICA}

El resto de este capitulo se relaciona con el tema de cómo esta distinción crucial puede llegar a constituir la base de los sistemas de cambio e intervención con valor práctico para la sociedad y el individuo. Yo mantengo que una teoria de la atribución, organizada según las líneas ya enunciadas de las distinciones filosóficas y legales básicas y de la lógica del lenguaje corriente, tiene un gran poder como esquema del que se pueden de- rivar medidas curativas, pero que este poder es por completo una consecuencia de la reorientación intelectual o conceptual que la teoría representa. Los temas empíricos, a excepción de los estudios cuyo único objetivo es evaluar el éxito de los sistemas curativos basados en la teoría, están más allá del ámbito de la teoría. Las "pruebas» empíricas de la teoría y los "refinamientos" de sus predicciones, basados en el laboratorio, constituyen una pista falsa en relación con el valor del conjunto de ideas que ha llegado a ser conocido como teoría de la atribución.

En la década pasada el papel de la experimentación en psicología social ha sufrido un asedio crítico desde posiciones muy diversas (p. ej., Gergen, 1978; Harré, 1980). La experimentación controlada ya no es el dios olímpico que fue. Sugiero que se puede prescindir de ella en los análisis de la teoría de la atribución, pero sin quitar mérito en ningún sentido al valor de la teoría como recurso práctico para mejorar la sociedad y ayudar al individuo. Ya que estoy más familiarizado con la literatura clínica, voy a utilizar la situación social que se da en el encuentro entre el terapeuta y el paciente como el punto de partida de esta exposición, pero los argumentos se pueden aplicar igualmente a otros campos, como el de la educación, donde un análisis de la teoría de la atribución resulta también relevante.

Consideremos un hipotético encuentro entre un terapeuta y un paciente. Una mujer angustiada ha buscado ayuda porque su marido la ha abandonado. Supongamos que el terapeuta conoce bien la teoría de la atribución y está deseando poner en práctica sus principios al tratar con sus pacientes. El terapeuta habla largamente con la mujer, ansioso de que ella le dé su versión de por qué se fue su marido. La paciente no es comunicativa, así que el terapeuta explora una serie de «atribuciones» hipotéticamente relevantes, utilizando para ello una lista de preguntas, cuya forma podria ser la siguiente. 


\section{Estudios}

1. ¿Cree usted que existia otra mujer? Si es así,

a. ¿Las relaciones sexuales entre ustedes eran buenas?

b. ¿Había una buena relación amistosa?

c. ¿Tenian muchas discusiones? $\mathrm{Si}$ es así, ¿sobre qué?... etc.

d. ¿Ha tenido usted a su vez amistad con algún otro hombre últimamente?... etc.

2. ¿Le regañaba mucho? $\mathrm{Si}$ es así, ¿sobre qué?... etc.

3. ¿Existía algo que él quisiera y que usted no podía darle?

4. ¿Cree usted que él ha perdido la cabeza de forma temporal?

5. ¿Ha estado él sometido a una fuerte tensión en el trabajo?... ¿Por cuestiones económicas?

Este es el tipo de preguntas que un terapeuta podría utilizar como «entradas" prospectivas al problema de un paciente. El objetivo al hacer estas preguntas es clasificar los pensamientos y reflexiones del paciente en diversas formas preconcebidas. Supongamos, después de varias sesiones, que el terapeuta se ha formado la impresión de que la paciente creía que su esposo la había abandonado porque, con el transcurso del tiempo, ella se había vuelto fría y distante con él, tratándole como un «mueble». Según el modelo de impotencia aprendida de Seligman, reformulada en términos de la teoria de la atribución (Abramson y otros, 1978), ella ha hecho una atribución «interna» (el origen del problema se identifica en ella misma) que es "global» (su actitud afectaba a una amplia gama de situaciones e individuos). La conclusión a partir del modelo reformulado de impotencia aprendida es que, en tales circunstancias, los déficits - su depresión y expectativa de impotencia futura - serán intensos y de larga duración.

Excluyendo la posibilidad de persuadir al esposo para que vuelva, la estrategia del terapeuta consistirá en "cambiar» algún aspecto de esta situación, y existen una serie de posibilidades que él o ella pueden intentar. Pueden intentar cambiar, o bien el valor estimado del esposo que se ha ido o, alternativamente, algún componente del esquema explicativo. Supongamos que el terapeuta valora cuál de estas dos estrategias funcionará mejor, rechaza la idea de intentar convencer a la mujer de que está mejor sin su esposo, ya que el conocimiento limitado que tiene de la relación puede hacer que este argumento sea dificil de vender, y se decide por la segunda opción: manipular el proceso de atribución en sí. Supongamos también que, en consultas posteriores con su cliente, el terapeuta se forma la impresión de que una gran parte del problema de éste es que se ha herido su amor propio; ella considera el abandono de su esposo como el resultado de un fracaso personal que atribuye a su vez a una caracteristica relativamente estable de su personalidad: su falta de afectuosidad. En este estadio el terapeuta intentará una crítica del razonamiento que la ha llevado a esta confusión, siguiendo quizá alguna de estas líneas:

1. Es normal que las relaciones entre las personas cambien de esta manera con el paso del tiempo (ella está aplicando criterios falsos para el éxito y el fracaso).

2. El que una relación deje de estar viva es algo que concierne a dos (p. ej., la causa es menos «interna» y más uexterna" que lo que ella cree).

3. La relación con este hombre puede haber terminado, pero siempre existe la posibilidad de relacionarse con otros hombres (p. ej., su frialdad es menos "global» y más «específica» que lo que ella cree).

4. Un periodo de separación es algo bueno, ya que la hará más sensible y extrovertida (p. ej., su frialdad es menos «estable» y más "temporal» que lo que ella cree).

De este ejemplo hipotético surgen dos cuestiones. La primera, específicamente relevante para la psicoterapia, es que la teoría de la atribución, aunque puede ser una parte útil e incluso necesaria de cualquier análisis de la situación terapéutica, 
no es en sí misma suficiente. El éxito y el fracaso, los determinantes de una autoestima alta o baja, están relacionados, por definición, con criterios socialmente derivados. Las atribuciones de éxito y fracaso implican una aceptación, o internalización, de tales criterios. Si los criterios que el individuo toma para si son absurdamente excéntricos o irrealmente rigidos, y tan exigentes que él nunca llega al nivel previsto, entonces las atribuciones de fracaso son, por así decirlo, "construidas dentro del sistema", y es bastante poco probable que, siguiendo estrictamente los principios de la atribución, pueda presentar el terapeuta un argumento convincente de que los ejemplos concretos de fracaso son resultado de factores externos, específicos o inestables. Intentar "cambiar" las atribuciones de un individuo en tales circunstancias seria forzar la credibilidad más allá de los límites razonables. Así, pues, una consideración fundamental al aconsejar debe ser la razonabilidad y normalidad de los criterios personales que el individuo se fija. Muy en la linea de esta observación están algunas fuentes de evidencia, que unen criterios de logro y moralidad disposicionalmente extremos con una tendencia a la enfermedad psíquica y también física (Blatt y otros, 1976; Glass, 1977; Totman, 1979 y en prensa) ${ }^{1}$.

Asi, la primera cosa a tener en cuenta sobre la aplicación de los principios de la teoría de la atribución a la terapia es que a menudo, y desde luego en los casos de amor propio herido, no son suficientes por sí mismos, y deben complementarse con un conocimiento detallado de la estructura normativa de la sociedad y de la "razonabilidad" de los objetivos del individuo. El terapeuta no sólo debe estar en posesión de una concepción de la sociedad elaborada de forma sistemática, sino que también debe reconocer el microcosmos social concreto en que se mueve el paciente, y apreciar sus propios objetivos y proyectos que están relacionados con estos sistemas de escala social más pequeños que sirven para estructu- rar su vida y marcar la dirección de sus esfuerzos. El éxito y fracaso sólo tienen sentido en relación con los criterios fijados, y aunque algunos de éstos podrian ser considerados de forma razonable como socialmente universales, o al menos ampliamente compartidos, es probable que la mayor parte de ellos no lo sean, y a menudo requieren una explicación y definición detalladas. Por lo tanto, gran parte de la terapia debe consistir en el intercambio de la información social, la «negociación» de diferentes mundos sociales y la confrontación entre las respectivas retóricas afines a estos mundos (cf. Harré, 1980). El etiquetaje por parte del atribucionista de lo quc dice un paciente no se puede hacer hasta que este intercambio negociador en dos direcciones esté bastante avanzado.

\section{La teoría de la atribución como relativista}

El segundo punto que se deriva de esta ilustración es más complejo: consiste en que el valor de la teoría de la atribución como un recurso terapéutico proviene fundamentalmente de su base filosófica. Lo que hace un terapeuta cuando está intentando llevar a la práctica los principios de la teoría de la atribución, en el tratamiento de individuos alterados, es escuchar las explicaciones que esa persona da sobre sus desgracias, hacer una unión teórica entre éstos y sus sintomas, e intentar intervenir cambiando algunos componentes de esta versión explicativa (una «atribución»), alterando por consiguiente el curso de la corriente cognitiva que, se supone, culmina en la reacción maladaptativa. $\mathrm{La}$ naturaleza esencialmente "neutral» o arbitraria, y su fundamento en una concepción muy relativista del hombre moral, es lo que constituye el sello del análisis de la atribución.

Consideremos el ejemplo señalado por Valins y Nisbett (1972) de un chico negro de 25 años, soltero, que llegó a la terapia profundamente preocupado porque pensaba que era homosexual. 
«Su atribución de la homosexualidad se basaba en varias observaciones. Su relación sexual era insatisfactoria, a menudo se encontraba mirando a la zona de los genitales de otros hombres, y él pensaba que su pene era más pequeño de lo normal. Esta última creencia parecía ser la principal causante de sus dificultades... Se inició la terapia explicándole las leyes ópticas - esto es, vistos desde arriba, los objetos en el mismo plano que la línea de visión, parecen más cortos. Se aconsejó al cliente que se mirara en un espejo y este procedimiento contribuyó a convencerle de que su pene, aunque no era de proporciones sobrehumanas, tenía un tamaño "normal". El terapeuta también le explicó que sus miradas hacia la zona de los genitales de otros hombres eran consecuencia natural de la idea que tenía de que su propio pene era más pequeño. Se persuadió así al cliente de que su conducta era una indicación de autoevaluación, y no de homosexualidad. Era "normal" que sintiera curiosidad por el tamaño de los penes de otros hombres. Por último, sus experiencias sexuales insatisfactorias se explicaron no como el resultado de un interés heterosexual insuficiente, sino como una consecuencia "normal" de su ansiedad ante una posible actuación inadecuada) (Valins y Nisbett, 1972, pp.138-139).

Al leer entre lineas este historial tenemos la impresión de que inteligentemente se ha dicho una «mentira piadosa». $\mathrm{El}$ plan para que se curara dependía de poder sustituir una creencia por otra, pero la autenticidad de las creencias no se pone en cuestión para nada, de hecho no se puede hacer sin alterar el carácter mismo del análisis de la teoría de la atribución. Para ser capaz de lanzarse a un programa de intervención, tanto en la terapia como en educación, el psicólogo debe considerar las creencias del sujeto o paciente como manipulables en principio, y para este fin es necesario que no tenga en cuenta el relativo valor de veracidad de las atribuciones que sus clientes hacen $o$ pueden ser inducidos a que hagan. En el primer ejemplo, debe sustituir la razón del abandono del marido por alguna otra que no sea la de la frigidez. En el ejemplo de Valins y Nisbett, el hecho de mirar los genitales a otros se le presentó al paciente como una actividad perfectamente normal y razonable. En ambos casos se ha hecho un "trabajo de venta" para persuadir al cliente de que acepte una explicación planificada, de forma más o menos arbitraria, en lugar de otra. La explicación sustitutiva es "arbitraria», en el sentido de que el único criterio que determina cuál es la explicación preferida por el terapeuta son las posibles consecuencias del cambio en el curso de la corriente cognitiva, y por consiguiente, en último lugar, aliviar los sintomas patológicos. La consideración de la veracidad o falsedad de las creencias particulares se exluye del análisis de forma característica y axiomática.

Leer este argumento como una crítica de la teoria de la atribución sobre bases éticas sería malinterpretar lo que aquí se pretende. El asunto en cuestión es que el valor de la teoría de la atribución, en la práctica, se deriva de un presupuesto, sobre el que se construye la teoría, acerca de cómo trabaja la mente humana al tratar los fenómenos sociales. En antiguos modelos de la acción humana, el panorama, en términos generales, era el de una auténtica razón por la que una acción se ejecutaba, y algunas otras razones concebibles las cuales eran falsas. Gradualmente, y en especial a través del trabajo conectado con la teoría de la disonancia cognitiva y el paradigma de la sumisión forzada, este concepto ha dado pie a la idea de que se pueden dar a la misma acción significados diferentes pero igualmente legitimos. Se puede explicar de diferentes formas alternativas pero igualmente auténticas.

En la situación de sumisión forzada, la disonancia se introduce de forma deliberada por el montaje experimental que se ha estructura sutilmente, de forma que una persona crea que está realizando una actividad (y experimentando por lo general alguna dificultad que no estaba prevista) no bajo presión, sino por su libre voluntad. El papel del experimentador consiste en introducir la libre elección en la situación de la forma más inteligente posible, y ya que el sujeto cree 
que está actuando libremente y no por presiones y obligaciones, de esta participación se pueden derivar consecuencias psicológicas y conductuales radicalmente diferentes de las que hubieran tenido lugar si él se hubiese visto a sí mismo obligado (ver, por ejemplo, Zimbardo, 1969; Totman, 1979).

En el diseño de estos experimentos estaba implícito un nuevo concepto de la acción, basado en el presupuesto de que no es apropiado representar la libre elección como una cualidad absoluta y no negociable. Es más útil concebirla como una percepción individual que varía sistemáticamente en condiciones diferentes, y que se puede manipular. Para que la disonancia surja de forma eficaz, el sujeto debe verse como persona que actúa por libre elección, pero es igualmente necesario que el experimentador, por otro lado, considere que la acción del sujeto emana de las presiones sociales, camufladas con cuidado a partir de la situación que él ha planificado deliberadamente. La cuestión, según el propósito del análisis, es que estas dos versiones se pueden considerar compatibles. El que el experimentador haga que el sujeto se comporte de una forma particular no invalida la propia versión del sujeto. La libre elección que el sujeto está experimentando no es una ilusión. Es la piedra angular de la teoría de la acción que él mismo construye, y su "teoria» se va a considerar igual de válida que la del experimentador. Las dos posiciones no se excluyen una a otra sino que son alternativas en el nuevo meta-nivel de análisis, en el que el centro se ha trasladado de la acción en sí a las percepciones y explicaciones que la gente hace de esa acción. Del mismo modo, la manipulación en los experimentos de la disonancia no se considera una violación de los niveles éticos. Es esta noción de la equivalencia verídica de las versiones de las personas sobre las acciones lo que está en el fondo de la teoría de la atribución y lo que constituye su principal innovación. Al aumentar un nivel del análisis, desde la explicación de la conducta y acción a la explicación de las explicaciones de la gente, el status axiomático de la investigación se transforma de forma fundamental. Además, se cambia de tal forma que las consideraciones éticas basadas en la antigua idea de la no equivalencia de las razones afirmadas es descalificada. No es que se rechacen de forma cruel e inhumana, sencillamente son irrelevantes. A través de lo que es en esencia un movimiento intelectual es posible empezar a hablar de "cambiar» las explicaciones que ofrece una persona. El logro de la teoría de la atribución, es por consiguiente, más de tipo filosófico - un «experimento de pensamiento"- que de tipo empiricista basado en el labratorio.

A primera vista, nos encontramos con un problema en este argumento. ¿Qué pasa con esos ejemplos en los que una versión dada es claramente débil, frente a una versión alternativa, viéndonos forzados a rechazarla como poco convincente? Los escritos sobre la teoria de la atribución están llenos de informes de «falsas atribuciones» que hacen los sujetos sobre los fenómenos. En el bien conocido estudio de Storms y Nisbett (1970), se indujo a los insomnes a atribuir los sintomas de su insomnio (aceleración del ritmo cardiaco, sucesión rápida de pensamientos, sudor, etc.) a los efectos secundarios de una pastilla que se les había dado como si fuese droga, pero que en realidad no era más que un placebo. Storms y Nisbett predijeron con éxito que ya que estos individuos estaban dotados de un medio "externo» de explicar los sintomas del insomnio, experimentarian menos emocionalidad y podrían quedarse dormidos con más rapidez que los sujetos control a los que también se les dio la misma pildora, pero a quienes se les dijo que ésta produciria efectos secundarios que no estaban relacionados con el insomnio. Al menos desde la perspectiva del experimentador es inequívoco: a los sujetos se les proporciona una explicación falsa de sus síntomas. Podemos decir que la explicación es falsa porque sabemos que los síntomas existian antes de que se les administrara 


\section{Estudios}

la pastilla y que probablemente persistirian despues de que se la retiren; no tiene por qué darse una relación entre pildora y sintomas. Pero este razonamiento sigue una directriz lógica o científica que los no científicos no necesariamente aplican de forma tan rigurosa $y$ consistente. De hecho, se puede presuponer con bastante seguridad que en el experimento de Storms y Nisbett los sujetos no resolvieron sus problemas de acuerdo con esta línea estrictamente racional. Si hubiera sido así, la predicción no se habría confirmado, ya que los sujetos no habrian aceptado la historia del experimentador sin ninguna crítica. Asi, pues, en un sentido, dentro de los términos de referencia de la forma no científica del análisis aplicado por los sujetos en este experimento, la creencia de que la pastilla causaba sintomas de insomnio es totalmente auténtica. Es auténtica en base a que el experimentador ha pasado información al sujeto, y el sujeto confia en que el experimentador le cuenta la verdad. Estos criterios que el sujeto usa para juzgar la validez de sus propias atribuciones son, en relación con su propio esquema social de las cosas - su propia "visión del mundo»-, suficientes.

La manipulación de la atribución en la terapia depende así de la explotación que se haga de las discrepancias entre dos esquemas sociales privados, constituidos de forma diferente, de dos puntos de vista sociales alternativos. Cuando un terapeuta se encuentra por primera vez con un paciente, hace todo lo posible por comprender a su cliente, utilizando el tiempo que sea necesario en la conversación. Pero, por hábil que pueda ser al comunicarse, por muy acertada que sea la posición de la otra persona para comprender y por muy global que sea la descripción del mundo social que puede construir, socialmente permanece distanciado del paciente en virtud de su repertorio diferente de normas sociales prescriptivas; sus valores, criterios, actitudes, ambiciones, proyectos objetivos y estilos son diferentes, algunos de los cuales, los más sobresalientes, están en función de su papel profesional en ese momento. Es precisamente en esta diferencia entre las personas, en sus respectivas gramáticas sociales, en donde se encuentra la potencialidad del cambio (tratamiento en este caso).

Todo lo que se necesita para apoyar este argumento es considerar las amplias diferencias que existen en las definiciones individuales de los términos abstractos cargados de valor, tales como "veracidad", «honestidad» y "amabilidad». Los Testigos de Jehová presentarán definiciones y casos ejemplificadores de estas cualidades muy diferentes de los freudianos; y los albañiles, de los ladrones, etc... "Veracidad» significa cosas muy diferentes en distintos contextos culturales y subculturales. Incluso subdivisiones más pequeñas de la sociedad, amigos íntimos, pandillas, colaboradores y otros grupos de referencia aportarán distinciones más sutiles. Mientras que la estructura de sus respectivos sistemas morales y sociales puede ser la misma, el "contenido» no. En todos los ejemplos señalados en este capitulo -el homosexual autoatribuido de Nisbett y Valins, los insomnes de Storms y Nisbett y mi ilustración de la mujer frígida - funciona la teoría basada en la atribución porque los pacientes (o sujetos) aluden a criterios para juzgar la aceptabilidad de lo que se les dice (la «manipulación» de la teoría de la atribución), que son diferentes de los criterios que el equipo del terapeuta o experimentador va a desarrollar. El principio regulador decisivo de este último es, hablando claro, el pragmatismo o utilitarismo, mientras que el del primero es la confianza. El dominio de la confianza sobre todas las cosas es propio del que busca ayuda y consejo; el dominio del pragmatismo, del que lo da. La viabilidad de todo el escenario, cuidadosamente estructurado, sujeto-experimentador y paciente-terapeuta, depende del mantenimiento mutuo de estas orientaciones rolregla claramente distintas e inequívocas durante el experimento o el curso del tratamiento. Todo el ritual que acompaña a la interacción, conjunto, escenario y 
apoyos de la clínica y laboratorio, ayuda a las dos posiciones a mantener esta división social tan fundamental para el resultado.

Así, pues, el terapeuta debe operar en dos niveles diferentes. Primero, como miembro, junto con el paciente, de la misma cultura, debe conseguir, si no lo posee ya de primera mano, un conocimiento excepcional de la constitución de la sociedad y de sus ramificaciones más esotéricas. En segundo lugar, como profesional, debe permanecer socialmente distanciado del paciente en lo referente a los tipos de explicación que él considera, y a los criterios regulativos - estandards - frente a los cuales se miden sus teorías. Estas son las cosas que definen su po sición como terapeuta. Su pragmatismo o utilitarismo se debe plantear a las claras frente a la receptividad y confianza del paciente. Sin este contraste, no existe potencial para la intervención basada en la teoria de la atribución. El ámbito de este contraste está en la institucionalización de la relación «paciente-terapeuta» de nuestra cultura.

El caso más agudo, y posiblemente más interesante, del tipo de encuentro que depende del mantenimiento de dos sistemas explicativos alternativos, se refiere a la situación que se plantea cuando la terapia se centra en la idea de conseguir que el paciente produzca acciones voluntarias autoiniciadas. Una gran proporción de las técnicas contemporáneas en psicoterapia, da especial relieve a las consecuencias curativas que se siguen de las conductas autoiniciadas, ocupándose de cómo conseguir que un paciente emita respuestas voluntarias o «asertivas». El modelo de la impotencia aprendida de Seligman sobre la depresión, identifica como causa de los episodios depresivos una uestructura cognitiva de impotencia», una creencia generalizada por parte del paciente de que sus respuestas no son efectivas para procurarse recompensas. Los programas terapéuticos son intentos de cambiar esta estructura, incluyendo manipulaciones que favorezcan las atribuciones de agencia (atribuciones in- ternas) por parte del paciente (Abramson y otros, 1978).

La idea utilizada en la terapia de conducta cognitiva de que la clave de la "reestructuración cognitiva» es conducta, y de que el cambio en los esquemas mentales de una persona se puede efectuar mejor a través de un planeamiento de nuevas formas conductuales, no es en conjunto nada nueva. Fue prefigurada y explotada (aunque descrita en una jerga diferente) en la teoría de la disonancia cognitiva, en el paradigma de la sumisión forzada y también, tal vez de la forma más directa de todas, en la teoría de la autopercepción de Bem (Bem, 1972) y su idea de que las actitudes toman su clave de la conducta. Todas estas formulaciones son desarrollos diferentes de una idea embrionaria muy similar, la de que la potencialidad para el cambio cognitivo depende del establecimiento y mantenimiento de una disparidad entre los criterios sociales del sujeto y del experimentador (o del paciente y el terapeuta), cuyo origen está en sus diferentes compromisos metafísicos. Esta diferencia cristaliza en los distintos pre-esquemas atributivos que están en la raíz del proceso terapéutico.

Tampoco, sospecho yo, es especialmente nueva en la terapia la idea del "entrenamiento asertivo» y las técnicas, derivadas de ella, para producir «respuestas» iniciadas voluntariamente. Se ha convertido casi en un cliché entre las distintas escuelas de terapia, incluyendo el consejo psiquiátrico, que al principio de las sesiones terapéuticas el terapeuta ponga de relieve a su paciente la importancia que le da a que éste juegue un papel activo en el tratamiento ( $(\ldots .$. la iniciativa real debe venir de usted»... «usted ya ha dado el paso más importante al venir aquí»... «todo lo que yo puedo hacer es indicarle la dirección correcta, pero es usted quien tiene que hacer el trabajo realmente»... etc.).

Inducciones de este tipo, mantenidas a lo largo del tiempo, contribuyen a fijar el escenario con respecto a las atribuciones prospectivas del paciente para las se- 
siones de tratamiento. Son directivas o ultimatums al paciente para que se prepare a hacer atribuciones «internas» de las acciones que llevará a cabo por orden del terapeuta. Este, sin embargo, si quiere ser efectivo en la producción de las conductas deseadas en el paciente, deberá ser poderoso, como corresponde a un terapeuta, debe mantener el compromiso de atribuir las acciones del sujeto a causas «externas», predominantemente él mismo. Es esta perpetuación de una discrepancia en los compromisos atributivos la que permite que haya resultados en los encuentros psicoterapéuticos, cuya finalidad es cambiar los recursos cognitivos del individuo, de forma que se encuentre mejor equipado para enfrentarse a las exigencias de su ambiente social. El paciente se ve necesariamente a sí mismo actuando libremente, mientras que a la vez el terapeuta ve las acciones del paciente como consecuencia de sus manipulaciones y recomendaciones. Por consiguiente, la reestructuración cognitiva necesaria se logra a través del tipo de mecanismos psicológicos descritos en las teorías de Festinger y Bem (véase también Totman, 1979) que dependen de forma crítica, para su activación, de las atribuciones de internalidad por parte del actor. En la teoria de Bem están resumidas en la frase: «las actitudes siguen a la conductam. En el contexto de nuestra discusión se podría extender y decir: «los esquemas mentales adaptativos se construyen a partir de actos (atribuidos internamente) contextualmente apropiados». Como he intentado mostrar, ni la versión del paciente ni la del terapeuta deberían de tomarse como autoridad. Desde el «meta-sistema» en cuyos términos es apropiado analizar la interacción, ambas versiones son igualmente válidas, y son perfectamente compatibles (véase Totman, 1980, para una ampliación de este tema).

En este análisis, pues, la terapia basada en la atribución depende de forma crucial de la coexistencia de esquemas atributivos discrepantes ${ }^{2}$, y la misma teoría de la atribución se basa en una meta-lógica de compatibilidad de estas posiciones alternativas. Ya no tiene sentido hablar de uerrores atributivos" y «falsas atribuciones». Hacer esto supone aceptar una versión medio hecha de la teoría de la atribución y cortar las alas de un elemento significativo dentro de un nuevo género de pensamiento sobre la conducta social que abandona el limitado empirismo basado en el laboratorio, y que ha dominado el tema desde hace tanto tiempo. Es una teoría "pura» con un considerable potencial de aplicación a los problemas sociales. Paradójicamente, puede ocurrir que cuanto mayor sea la ruptura con el empirismo clásico, más poderosa sea una teoría psicológica como recurso para afrontar los problemas sociales en un nivel práctico.

\section{Notas}

1 Abramson y otros (1978), en su reformulación del modelo de la impotencia aprendida, reconocen la posibilidad de que haya dos tipos diferentes de depresión, sólo uno de los cuales se caracterice por la baja autoestima. Es interesante que la moralidad y normas excesivamente elevadas parezcan estar asociadas con la depresión en la que los déficits de autoestima son prepotentes.

2 Hasta qué punto se puede decir lo mismo de otras formas de psicoterapia, especialmente el consejo psiquiátrico, es una cuestión interesante y sugerente.

\section{Referencias}

Abramson, L. Y., Seligman, M. E. P. y Teasdale, J. D. Learned helplessness in humans: critique and reformulation. Journal of Abnormal Psychology. 1978. 87, 49-74.

Bem, D. J. Self-perception theory. En L. Berkowitz (Ed.). Advances in experimental social psychalogy (vol. 6) pp.1-62. New York: Academic Press. 1972.

Blatt, S. J., D'AFFlitTi, J. P., y Quinlan, D. M. Experiences of depression in normal young adults. Journal of Abnormal Psychology. 1976. 85, 383-389. 
FEstinger, L. A theory of cognitive dissonance. Evanston, Ill.: Row Peterson, 1957.

GERGEN, K. Toward generative theory. Journal of Personality and Social Psycbology. 1978. 36, 1344-1360.

Glass, D. C. Bebaviour Pattern, Stress and Coronary Disease. New York: McGraw-Hill, 1977.

HARrÉ, R. Social Being: a Tbeory for Social Psycbology. Oxford: Blackwell, 1980.

LANGER, E. J. Rethinking the role of thought in social interaction. En J. H. Harvey, W. Ickes y R. F. Kidd (Eds.) New Directions in Attribution Researcb (vol. 2) pp.35-38. New Jersey: Erlbaum, 1978.

STORMS, M. D. y NisBETT, R. E. Insomnia and the attribution process. Journal of Personality and Social Psychology. 1970. 16, 319-328.

TOTMAN, R. G. An approach to cognitive dissonance theory in terms of ordinary language. Journal for the Theory of Social Bebaviour. 1973. 3, 215-238.

Totman, R. G. Social Canses of Illness. London: Souvenir Press (y New York: Pantheon). 1979.

TOtman, R. G. The incompleteness of ethogenics. European Journal of Social Psycbology. 1980. 10, $17-41$.

Totman, R. G. Psychosomatic theories. En J. R. Eiser (Ed.) Social Psycbology and Behavioural Medicine. London: Wiley (en prensa).

VALINS, S. y NisBetT, R. E. Attribution processes in the development and treatment of emotional disorders. En E. E. Jones, D. E. Kanouse, H. H. Kelley, R. E. Nisbett, S. Valins y B. Weiner (Eds.) Attribution: Perceiving the Causes of Bebaviour. pp.137-149. New Jersey: General Learning Press. 1972.

Zimbardo, P. G. The Cognitive Control of Motivation. Illinois: Scott, Foresman. 1969. 